\title{
Aprovechamiento de la naranjilla (Solanum quitoense Lam.) variedad INIAP Quitoense-2009 para la obtención de una bebida carbonatada
}

\section{Use of the naranjilla (Solanum quitoense Lam.) variety INIAP Quitoense-2009 to obtain a carbonated beverage}

\author{
Juan Gaona-Gonzaga' ${ }^{1}$ Diego Montesdeoca-Espin' ${ }^{1}$, Beatriz Brito-Grandes ${ }^{2}$, \\ Andrea Sotomayor-Correa ${ }^{2}$, William Viera-Arroyo ${ }^{2}$
}

\begin{abstract}
Resumen
Se elaboró una bebida carbonatada con pulpa natural de naranjilla (variedad INIAP Quitoense 2009). Para ello se realizaron combinaciones con tres concentraciones de pulpa (15,20, $25 \%$ ), tres concentraciones del edulcorante natural stevia $(10,12,14 \%)$ y agua, registrándose variables de sólidos solubles, pH y acidez titulable. Se determinó la mejor formulación mediante el análisis de varianza, prueba de Tukey al 5 \% y la aceptación mediante el análisis sensorial. Posteriormente, se adicionó $0.4,0.5$ y 0.6 \% de estabilizante (goma arábiga) y se procedió a medir los mismos parámetros y la altura de sedimentación. Los resultados se analizaron estadísticamente. Para la carbonatación se pasteurizó y se envasó la bebida más estable en botellas de vidrio ámbar de $350 \mathrm{ml}$ y se agregó $1.8 \mathrm{~g}$ de $\mathrm{CO}_{2}$ sólido (hielo seco). Se selló el envase y se realizaron las pruebas sensoriales después de 24 horas para determinar la aceptación de la bebida carbonatada. La formulación más aceptable fue: 25 \% pulpa, 10 \% edulcorante, 0.5 \% estabilizante, 0.1 \% benzoato de sodio, $0.05 \%$ ácido cítrico, $0.5 \% \mathrm{CO}_{2}$ sólido y el resto agua. La pulpa de naranjilla puede ser utilizada en la elaboración de una bebida carbonatada, ya que cumple con la normativa ecuatoriana.
\end{abstract}

\section{Palabras clave}

Jugo; gasificado; fruta.

\begin{abstract}
A carbonated beverage was made with natural naranjilla pulp (INIAP Quitoense 2009 variety). Combinations were made with three pulp concentrations $(15,20,25 \%)$, three concentrations of the natural sweetener stevia $(10,12,14$ $\%)$ and water, registering variables of soluble solids, $\mathrm{pH}$ and titratable acidity. The best formulation was determined by analysis of variance, Tukey test at $5 \%$ and acceptance by sensory analysis. Subsequently, $0.4,0.5$ and $0.6 \%$ stabilizer (gum arabic) and the same parameters and sedimentation height were measured. Results were analyzed statistically. For carbonation, the most stable beverage was pasteurized and bottled in $350 \mathrm{~mL}$ amber glass bottles and $1.8 \mathrm{~g}$ of solid $\mathrm{CO}_{2}$ (dry ice) was added. The container was sealed and sensory tests were after 24 hours to determine the acceptance of the carbonated beverage. The most acceptable formulation was: $25 \%$ pulp, $10 \%$ sweetener, $0.5 \%$ stabilizer, $0.1 \%$ sodium benzoate, $0.05 \%$ citric acid, $0.5 \%$ solid $\mathrm{CO}_{2}$ and water. The naranjilla pulp can be used in the elaboration of a carbonated beverage, since it fulfills the Ecuadorian regulations.
\end{abstract}

\section{Keywords}

Juice; gasified; fruit

\section{Introducción}

Las bebidas carbonatadas son las más consumidas en el mundo, según datos registrados en el informe anual que presenta la industria alimenticia, América se ubica como el principal consumidor con el 39 \%. La población joven entre los 12 a 19 años registra un consumo del 81 \%,

\footnotetext{
$1 \quad$ Universidad Central del Ecuador, Quito, Ecuador \{juanpaiq@hotmail.com, dmontesdeoca@uce.edu.ec\}

2 Instituto Nacional de Investigaciones Agropecuarias, Quito, Ecuador \{beatriz.brito, andrea.sotomayor, william.viera\}@iniap. gob.ec
} 
siendo este el grupo que más consume este tipo de bebidas (Silva y Durán, 2014). En el Ecuador, el consumo per cápita de bebidas carbonatadas es de 50 l/año (INEC, 2015). Debido a la alta demanda de este tipo de bebidas existe el interés de desarrollar investigaciones para la elaboración de productos similares que aporten valor nutritivo, generando más alternativas en el mercado. Actualmente, en el mercado nacional no existe una bebida carbonatada de pulpa de naranjilla, razón por la cual esta fruta constituye una alternativa de industrialización para generar valor agregado. Por otro lado, el Ecuador cuenta con una producción aproximada de 28 000 t/año de naranjilla, siendo esta fruta de gran importancia económica para las comunidades indígenas y pequeños productores nacionales (Revelo et al., 2010).

La naranjilla (Solanum quitoense Lam.) posee un importante contenido de vitaminas A, B y $C$, además de generar un aporte importante en calcio y hierro posee propiedades antioxidantes (Andrade-Cuvi et al., 2015), en este sentido se han estudiado las características físico-químicas de la variedad INIAP Quitoense 2009 (Viteri et al., 2009; Brito et al., 2012) donde se ha identificado el contenido de vitamina $\mathrm{C}$, carotenoides y compuestos fenólicos, macro y micro minerales, determinando que es un fruto con excelente potencial nutricional e industrial, ya que posee una gran aceptación en los consumidores, por lo cual se fomenta su consumo.

La variedad INIAP Quitoense 2009 presenta parámetros de calidad como: rendimiento de pulpa (58.86 \%), sólidos solubles (10.80Brix), acidez titulable (2.56 \% ácido cítrico), pH (3.00), el color de la pulpa (verde) tiene un bajo incremento del índice de oscurecimiento (5.64 \% en una hora) siendo la oxidación mínima que junto con el tamaño grande del fruto y la calidad nutricional, le dan un excelente atributo para ser considerada como materia prima para la industria, como la elaboración de bebidas frutales y muy atractiva para diferentes usos en la agroindustria (Brito et al., 2012).

La mayor parte de la producción de naranjilla se comercializa como fruta fresca, existiendo diversas alternativas de valor agregado.Esta investigación permitió elaborar una bebida carbonatada a partir de la pulpa de naranjilla, al haberse determinado la relación entre los diferentes componentes de la formulación, la caracterización física, química y sensorial, que conserva la identidad básica de la fruta utilizada.

\section{Materiales y métodos}

\subsection{Materia prima}

Se utilizó como materia prima la variedad mejorada de naranjilla (Solanum quitoense Lam.) variedad INIAP Quitoense 2009, proporcionada por el Programa de Fruticultura de la Estación Experimental Central Amazonia del INIAP, ubicada en el cantón Joya de los Sachas.

Además, en este proceso se usó el edulcorante natural stevia adquirido del proveedor local Stevida/Ecuador; estabilizante goma arábiga, ácido cítrico y benzoato de sodio de calidad alimentaria que se lo adquirió en la Casa del Químico. El dióxido de carbono sólido conocido como hielo seco se adquirió a un distribuidor artesanal y agua embotellada.

\subsection{Procedimiento}

Para la obtención de la pulpa se utilizó 20 kg de fruta fresca y se realizó la formulación, estabilización, pasteurización, carbonatación hasta el envasado, como se detalla en la Figura 1. 
Figura 1. Proceso de elaboración de una bebida carbonatada de naranjilla

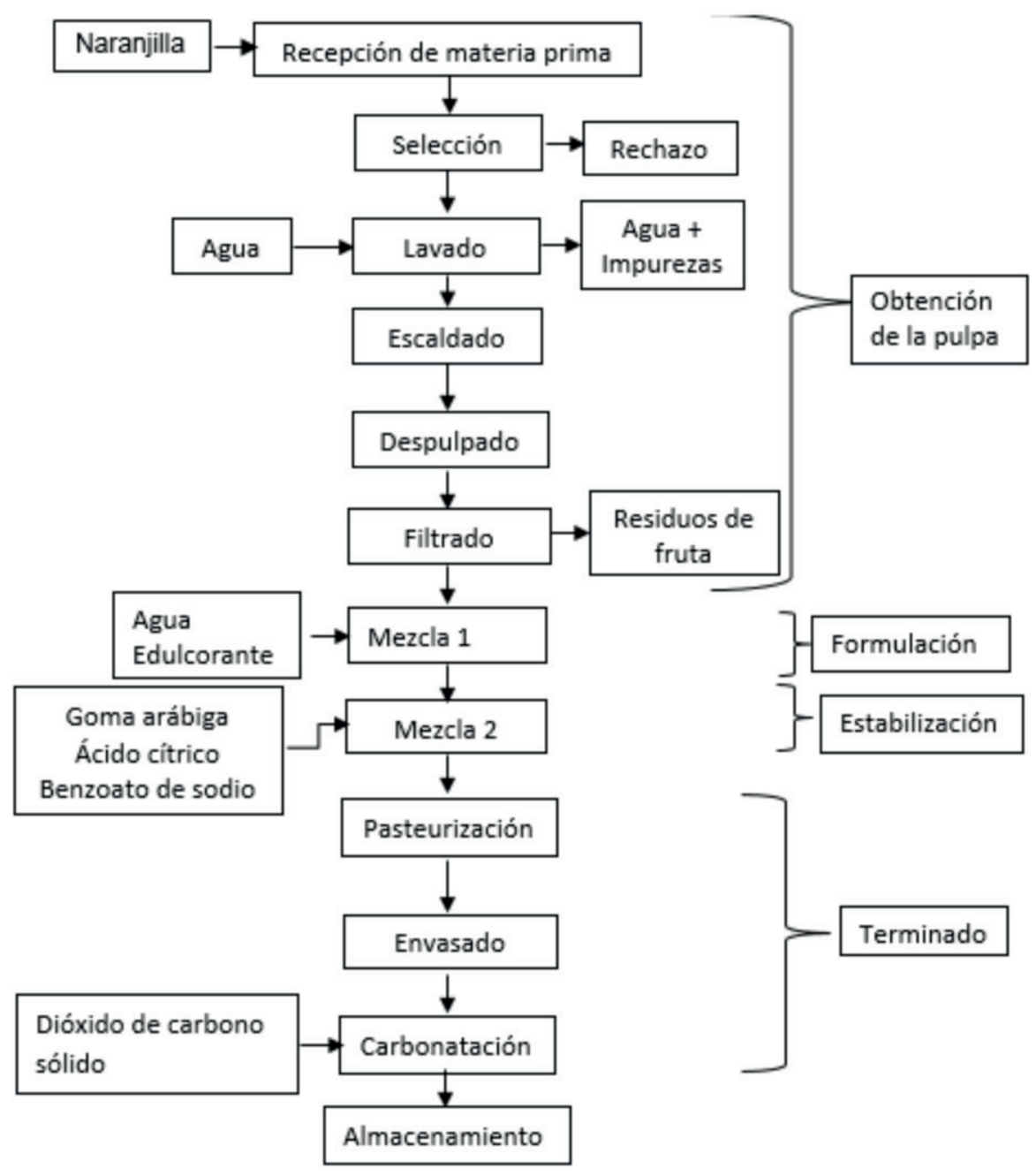

\subsubsection{Descripción del proceso}

Selección: La fruta fue seleccionada con un índice de madurez de cuatro, según la escala de madurez establecida por el Instituto Nacional de Investigaciones Agropecuarias (INIAP) (Brito et al., 2012), descartando aquellas que se encuentren en mal estado, estropeadas y que no cumplan con la madurez antes mencionada.

Lavado: Se realizó por inmersión con agua potable, con el uso de una esponja se procedió a refregar la superficie de la fruta, con la finalidad de eliminar cualquier impureza presente como restos de hojas, tierra y pequeñas vellosidades.

Escaldado: Este proceso térmico consistió en la inmersión de la fruta en agua a una temperatura promedio de entre 85 y $92^{\circ} \mathrm{C}$ por un tiempo de 10 minutos, posteriormente la fruta fue enfriada rápidamente con agua a $10^{\circ} \mathrm{C}(\mathrm{FAO}, 2013)$. La finalidad de este proceso fue inactivar las enzimas que se encuentran presentes en la fruta y que serían las responsables de generar una oxidación en el producto final. 
Despulpado: Para esta etapa del proceso se utilizó una licuadora doméstica marca Oster. Se procedió a licuar y, posteriormente, a filtrar con una cernidora la pulpa de la fruta térmicamente tratada. Además, se midió el pH y los sólidos solubles, con la finalidad de cumplir con los requisitos establecidos en la norma técnica ecuatoriana NTE INEN 2337-2008, que establece un valor máximo de $\mathrm{pH}$ de 4.5 y un valor mínimo de sólidos solubles $6.0{ }^{\circ} \mathrm{Brix}$. Luego de verificarse que la pulpa de naranjilla cumplía con la norma mencionada, se procedió a colocar en fundas herméticas de $150 \mathrm{~g}$, las que se conservaron en congelación.

Formulación de la bebida de naranjilla: Se realizó la formulación con base a tres concentraciones de pulpa $(15,20$ y $25 \%)$ y del edulcorante stevia $(10,12,14 \%)$, se evaluaron los parámetros sólidos solubles ( ${ }^{\circ}$ Brix), acidez titulable (\% ácido cítrico) y el pH. La norma técnica ecuatoriana NTE INEN 2337-2008, para las bebidas de frutas, establece el contenido mínimo de pulpa del $10 \%$. Las formulaciones luego fueron sometidas a un análisis sensorial, con 30 panelistas no entrenados, en edades comprendidas entre 20-25 años, se utilizó la escala dando un valor de 1 (no me gusta muchísimo) a 7 (me gusta muchísimo) para los parámetros del sabor, color, olor y dulzor.

Estabilización: En esta etapa se utilizó la mejor combinación de pulpa y edulcorante, habiéndose evaluado la adición de distintos porcentajes de goma arábiga $(0.4,0.5$ y 0.6 \%) como estabilizante. Se estableció una dosis baja y otra alta, tomando como valor referencia el contenido del estabilizante usado en el estudio de Naddaf et al. (2012). La estabilidad se determinó, registrando la altura de la cama de sedimentación cada 8 horas, por un período de 48 horas.

Pasteurización: Se realizó con la finalidad de eliminar cualquier microorganismo que esté presente en el producto mediante el calentamiento hasta una temperatura de $70{ }^{\circ} \mathrm{C}$, durante 3 min y, posteriormente, se enfrió a una temperatura de $10{ }^{\circ} \mathrm{C}$ (Villareal et al., 2013).

Envasado: En esta etapa se utilizaron envases de vidrio color ámbar de 350 ml para evitar que se produzca la oxidación de la bebida, lo que afectaría su calidad.

Carbonatación: Para esta etapa se enfrió la bebida hasta una temperatura de $8{ }^{\circ} \mathrm{C}$ y se agregó 2.5 volúmenes de dióxido de carbono en estado sólido (Maselli y Dierking, 2018). Se selló el envase y se conservó en refrigeración a $5 \pm 2{ }^{\circ} \mathrm{C}$, durante 24 horas.

Almacenamiento: La bebida carbonatada se almacenó durante ocho días en refrigeración a una temperatura de $5 \pm 2{ }^{\circ} \mathrm{C}$.

Evaluación de la calidad: Se realizó el análisis nutricional en el producto final.

\subsection{Diseño experimental}

Para la formulación de la bebida, los factores en estudio fueron el contenido de pulpa $(15,20$ y $25 \%$ ) y el contenido de edulcorante (10, 12 y $14 \%$ stevia). Se formularon nueve combinaciones (tratamientos) resultantes de la interacción de los factores en estudio. Se utilizó un diseño completamente al azar (DCA), con un arreglo factorial $3^{2}$ con tres repeticiones.

Se evaluaron las siguientes variables de respuesta: $\mathrm{pH}$ con un potenciómetro marca Mettler Toledo. Sólidos solubles se utilizó un refractómetro digital marca Mettler Toledo reportándose como grados Brix. Acidez titulable se siguió el procedimiento establecido en la norma NTE INEN 1091-2008. Los datos generados se analizaron estadísticamente mediante un análisis de varianza (ANOVA), con el uso de la prueba de Tukey al $5 \%$ para establecer diferencias entre las medias. Se utilizó el paquete estadístico INFOSTAT versión 2017. 
Para la etapa de estabilización, se evaluaron los contenidos de estabilizante $(0.4,0.5$ y 0.6 \% goma arábiga), se registraron las mismas variables de la etapa de formulación, para ello se utilizó al azar un diseño con tres repeticiones y el análisis estadístico.

Análisis sensorial: Se utilizó la escala desarrollada por el Departamento de Nutrición y Calidad del INIAP, con 30 panelistas no entrenados. Se evaluaron los parámetros del sabor, color, olor y dulzor. Los datos generados se analizaron estadísticamente mediante un análisis de varianza (ANOVA) y a través de la prueba de Tukey al $5 \%$ para establecer diferencias entre las medias.

\section{Resultados y discusión}

\section{1. Análisis de la formulación, estabilización y aceptabilidad del producto}

La fruta presentó un contenido de sólidos solubles de $10.85^{\circ} \mathrm{Brix}, 2.56$ \% de ácido cítrico, con un índice de madurez de 4.22 y un color amarillo anaranjado. El contenido de azucares totales es de $4.62 \%$, la vitamina C $53.33 \mathrm{mg} / 100 \mathrm{~g}$, polifenoles totales $0.81 \mathrm{mg} / \mathrm{g}$, carotenoides totales $1.27 \mu \mathrm{g} / \mathrm{g}$, calcio $48 \mu \mathrm{g} / \mathrm{g}$, potasio $3090 \mu \mathrm{g} / \mathrm{g}$.

En la Tabla 1 se puede observar los resultados de la formulación del producto, en donde el contenido de sólidos solubles aumenta conforme se aumenta el contenido del edulcorante, habiéndose encontrado diferencias no significativas. Se comparó los sólidos solubles con los establecidos en la norma técnica ecuatoriana INEN 1101-2008 que establece que una bebida debe poseer como mínimo un valor mayor a $7^{\circ} \mathrm{Brix}$, para poder ser considerada como bebida gaseosa, todos los tratamientos cumplen con este requisito.

En el pH no se observaron diferencias significativas, sin embargo, alcanzó un valor promedio de 3.32 el cual se ajusta con las normativas, que establecen un valor de 5.0 como máximo para bebidas gaseosas.

La variable acidez titulable reportada como porcentaje de ácido cítrico, presentó diferencias estadísticas, donde el tratamiento con $25 \%$ de pulpa $+10 \%$ de edulcorante obtuvo una media de $0.50 \%$, siendo este valor el menor de todos los tratamientos y es considerado como el máximo para una bebida gaseosa. García-Mogollon et al. (2015), en su investigación utilizó la pulpa de maracuyá y reportó valores mayores de $0.5 \%$, justificando que por tratarse de frutas que poseen una acidez alta, como es el caso de la naranjilla.

Tabla 1. Resultados del análisis en la formulación de la bebida carbonatada

\begin{tabular}{|l|l|l|l|}
\hline \multicolumn{1}{|c|}{$\begin{array}{c}\text { Combinación } \\
\%\end{array}$} & \multicolumn{1}{|c|}{$\begin{array}{c}\text { Sólidos Solubles } \\
{ }^{\circ} \text { Brix }\end{array}$} & \multicolumn{1}{c|}{ pH } & \multicolumn{1}{c|}{$\begin{array}{c}\text { Acidez titulable } \\
\% \text { ácido cítrico }\end{array}$} \\
\hline $25 \%$ pulpa $+10 \%$ edulcorante & $10.45 \pm 0.26$ & $3.30 \pm 0.17$ & $0.51 \pm 0.04 \mathrm{~A}$ \\
\hline $25 \%$ pulpa $+12 \%$ edulcorante & $10.54 \pm 1.28$ & $3.29 \pm 0.19$ & $0.52 \pm 0.06 \mathrm{~A}$ \\
\hline $25 \%$ pulpa $+14 \%$ edulcorante & $10.63 \pm 0.75$ & $3.31 \pm 0.19$ & $0.55 \pm 0.02 \mathrm{~A} \mathrm{~B}$ \\
\hline $20 \%$ pulpa $+10 \%$ edulcorante & $10.57 \pm 0.60$ & $3.30 \pm 0.0 .18$ & $0.55 \pm 0.02 \mathrm{~A} \mathrm{~B}$ \\
\hline $20 \%$ pulpa $+12 \%$ edulcorante & $10.78 \pm 0.84$ & $3.30 \pm 0.17$ & $0.63 \pm 0.04 \mathrm{~B}$ \\
\hline $20 \%$ pulpa $+14 \%$ edulcorante & $10.89 \pm 1.39$ & $3.31 \pm 0.19$ & $0.56 \pm 0.03 \mathrm{~A} \mathrm{~B}$ \\
\hline $15 \%$ pulpa $+10 \%$ edulcorante & $10.40 \pm 0.20$ & $3.42 \pm 0.16$ & $0.56 \pm 0.02 \mathrm{~A} \mathrm{~B}$ \\
\hline $15 \%$ pulpa $+12 \%$ edulcorante & $10.50 \pm 0.22$ & $3.43 \pm 0.18$ & $0.56 \pm 0.02 \mathrm{~A} \mathrm{~B}$ \\
\hline $15 \%$ pulpa $+14 \%$ edulcorante & $11.05 \pm 1.25$ & $3.32 \pm 0.18$ & $0.59 \pm 0.00 \mathrm{~A} \mathrm{~B}$ \\
\hline
\end{tabular}


El análisis de varianza durante el proceso de estabilización con el uso de la goma arábica se presenta en la Tabla 2, se muestra que no existen diferencias significativas en las variables de sólidos solubles y acidez titulable, pero sí existen diferencias para la variable del pH, donde el 0.5 y 0.6 \% de goma arábica presentan una media de 3.53 para el valor del pH.

En la variable altura de sedimento, se definió el contenido de goma arábica siendo del 0.5 \% el que generó menor sedimentación en la bebida, este resultado concuerda con lo mencionado por Gracia (1998), quien recomienda utilizar bajas concentraciones de este estabilizante para no alterar las variables fisicoquímicas.

Tabla 2. Resultados de la estabilización de la formulación más adecuada

\begin{tabular}{|l|l|l|l|l|}
\hline \multicolumn{1}{|c|}{ Tratamiento } & \multicolumn{1}{|c|}{$\begin{array}{c}\text { Sólidos Solubles } \\
{ }^{\circ} \text { Brix }\end{array}$} & \multicolumn{1}{|c|}{$\mathbf{p H}$} & \multicolumn{1}{|c|}{$\begin{array}{c}\text { Acidez } \\
\% \text { ácido cítrico }\end{array}$} & $\begin{array}{c}\text { Altura sedimento } \\
\mathbf{M m}\end{array}$ \\
\hline $0.4 \%$ goma arábiga & $10.35 \pm 0.13$ & $3.50 \pm 0.003 \mathrm{~B}$ & $0.55 \pm 0.04$ & $>1.5$ \\
\hline $0.5 \%$ goma arábiga & $10.11 \pm 0.41$ & $3.53 \pm 0.001 \mathrm{~A}$ & $0.55 \pm 0.06$ & $>1.0$ \\
\hline $0.6 \%$ goma arábiga & $10.21 \pm 0.12$ & $3.53 \pm 0.02 \mathrm{~A}$ & $0.57 \pm 0.05$ & 2 \\
\hline
\end{tabular}

En los resultados del análisis sensorial presentados en la Tabla 3, se observa que la bebida carbonatada de pulpa de naranjilla tuvo una aceptación favorable en todos los parámetros evaluados, resultados que concuerdan con la investigación realizada por Silva y Durán (2014), quienes expresan que la naranjilla cuenta con una gran aceptación por los consumidores, principalmente por el sabor y el olor.

Tabla 3. Resultados del análisis de aceptación de la bebida carbonatada

\begin{tabular}{|l|c|c|c|c|}
\hline \multicolumn{1}{|c|}{ Parámetro } & $\begin{array}{c}\text { Sabor } \\
\%\end{array}$ & $\begin{array}{c}\text { Color } \\
\%\end{array}$ & $\begin{array}{c}\text { Olor } \\
\%\end{array}$ & $\begin{array}{c}\text { Dulzor } \\
\%\end{array}$ \\
\hline No me gusta muchísimo & 0 & 0 & 0 & 3.3 \\
\hline No me gusta mucho & 10 & 3.3 & 3.3 & 20 \\
\hline No me gusta ni me disgusta & 16.7 & 33.3 & 40 & 26.7 \\
\hline Me gusta mucho & 33.3 & 43.4 & 33.3 & 36.7 \\
\hline Me gusta muchísimo & 40 & 20 & 23.4 & 13.3 \\
\hline
\end{tabular}

La composición final de la bebida carbonatada se describe en la Tabla 4. Además de los factores evaluados (pulpa, edulcorante y estabilizante), se adicionó benzoato de sodio y ácido cítrico en pequeñas cantidades como coadyuvantes alimentarios para el proceso, que como aditivos alimentarios son permitidos por el Código de la Alimentación, los cuales desempeñan el papel de conservar el producto, incrementando el tiempo de almacenamiento de la bebida (CODEX, 2018).

Las bebidas gaseosas comerciales utilizan saborizantes, colorantes y demás aditivos artificiales para la formulación de sus productos, lo cual representa una ventaja para la bebida carbonatada de naranjilla, debido a que en esta investigación no se utilizó saborizantes ni colorantes artificiales, obteniéndose el sabor y el color de la bebida por el uso de la pulpa natural propia de la fruta de naranjilla. Actualmente en el mercado existe ocho edulcorantes no calóricos permitidos por la FDA (U.S. Food \& Drug Administration), sin embargo solo uno es de origen natural conocido como stevia que es 200 a 400 veces más dulce que el azúcar de caña y se utilizó en esta investigación. 
Tabla 4. Composición de la bebida carbonatada final

\begin{tabular}{|l|c|}
\hline \multicolumn{1}{|c|}{ Componentes } & Porcentaje (\%) \\
\hline Agua & 63.8 \\
\hline Pulpa de naranjilla & 25.0 \\
\hline Edulcorante & 10.0 \\
\hline Goma arábiga & 0.5 \\
\hline Hielo seco & 0.5 \\
\hline Benzoato de sodio & 0.1 \\
\hline Ácido cítrico & 0.1 \\
\hline
\end{tabular}

\subsection{Análisis nutricional de la bebida carbonatada}

Se realizó el análisis nutricional a la bebida carbonatada de pulpa de naranjilla, presentado en la Tabla 5, se muestra que esta bebida proporciona beneficios a la salud del consumidor por el bajo contenido de edulcorante $(8.33 \mathrm{mg} / \mathrm{ml})$ y de sodio $(0.083 \mathrm{mg} / \mathrm{ml})$, compuestos que son altos en otras bebidas gaseosas comunes y que generalmente presentan un alto contenido de azúcares (hasta $398 \mathrm{mg} / \mathrm{ml}$ de este edulcorante) y el contenido de sodio $(0.230 \mathrm{mg} / \mathrm{ml}$ ).

Tabla 5. Resultados del análisis nutricional de la bebida carbonatada final

\begin{tabular}{|c|c|c|}
\hline \multicolumn{3}{|c|}{ Tamaño por porción 240 ml } \\
\hline \multicolumn{3}{|l|}{ Porciones por envase 1} \\
\hline \multicolumn{3}{|l|}{ Cantidad por porción } \\
\hline ENERGIA (Calorías) & $377 \mathrm{~kJ}$ & 90 (cal) \\
\hline Energías por grasa (Calorías de Grasa) & $0 \mathrm{~kJ}$ & 0 (cal) \\
\hline \multicolumn{3}{|c|}{$\%$ valor diario* } \\
\hline Grasa Total & $0 \mathrm{~g}$ & $0 \%$ \\
\hline ácidos grasos saturados & $\mathrm{Og}$ & $0 \%$ \\
\hline ácidos grasos-trans & $0 \mathrm{~g}$ & \\
\hline ácidos grasos monoinsaturados & $\mathrm{Og}$ & \\
\hline ácidos grasos poliinsaturados & $0 \mathrm{~g}$ & \\
\hline Colesterol & $0 \mathrm{mg}$ & $0 \%$ \\
\hline Sodio & $20 \mathrm{mg}$ & $1 \%$ \\
\hline Carbohidratos totales & $22 \mathrm{~g}$ & $7 \%$ \\
\hline Azúcares & $2 \mathrm{~g}$ & \\
\hline Proteína & $0 \mathrm{~g}$ & $0 \%$ \\
\hline
\end{tabular}

* Los porcentajes de los valores diarios están basados en una dieta de 8380 kJ (2 000 calorías)

\section{Conclusiones y recomendaciones}

Se determinó que la formulación de: 25 \% pulpa de naranjilla, 10 \% edulcorante stevia y 0.5 \% estabilizante goma arábiga, fue la más adecuada para elaborar una bebida carbonatada de pulpa de naranjilla. Esta formulación cumplió con los parámetros de los sólidos solubles, pH, porcentaje de $\mathrm{CO}_{2}$ y de pulpa, que son establecidos por la norma técnica ecuatoriana NTE INEN 
1101-2008, que establece los parámetros mínimos que debe cumplir un producto para poder ser considerado y comercializado como una bebida gaseosa. Además, que la bebida desarrollada, posee niveles bajos tanto de azúcar como de sodio, resultando beneficiosa para la salud.

Se recomendaría investigar nuevas formulaciones con otras frutas, y la combinación de las mismas, para generar de esta manera nuevas alternativas que sean apetecidas por los potenciales consumidores, que generen competitividad con las bebidas gaseosas existentes actualmente en el mercado.

\section{Bibliografía}

Andrade, M., Moreno, C., Guijarro, M., Concellón, A. (2015). Caracterización de la naranjilla (Solanum quitoense) común en tres estados de madurez. Revista Iberoamericana de Tecnología Postcosecha, 16 (2): 215-221.

Brito, B., Espin, S., Vásquez, W., Viteri, P., López, P., Jara, J. (2012). Manejo poscosecha, características físicas y nutricionales de la naranjilla para el desarrollo de pulpas y deshidratados. Quito, Ecuador: INIAP.

CODEX. (2018). Norma General para los Aditivos Alimentarios CODEX STAN 192-1995. Roma: FAO.

FAO. (2013). Fichas Técnicas. Procesado de Frutas. Recuperado de http://www.fao.org/3/a-au168s.pdf (accedido el 18/10/2018).

García-Mogollón, C., Alvis-Bermudez, A., Romero, P. (2015). Aplicación del Mapa de Preferencia Externo en la Formulación de una Bebida Saborizada de Lactosuero y Pulpa de Maracuyá. Información tecnológica, 26(5), 17-24.

Gracia, O. (1998). La goma arabiga: Una maravilla natural que nos beneficia a todos. Industria Alimentaria, 1-12. INEC. (2015). Revista de estadísticas y metodologías. Vol. 1. № 1. Recuperado de http://www.ecuadorencifras.gob.ec/ingreso-y-consumo/ (accedido el 18/10/2018).

INEN. (2008). NTE INEN 1091. Bebidas Gaseosas. Requisitos. Recuperado de https://archive.org/details/ ec.nte.1101.2008/page/n1(accedido el 18/10/2018).

INEN. (2008). NTE INEN 1101. Bebidas gaseosas determinación de la acidez titulable. Recuperado de https://archive.org/details/ec.nte.1091.1984 (accedido el 18/10/2018).

INEN. (2008). NTE INEN 2337. NTE INEN 2337: Jugos, pulpas, concentrados, néctares, bebidas de frutas y vegetales. Requisitos. Recuperado de https://archive.org/stream/ec.nte.2337.2008\#page/n0 (accedido el 18/10/2018).

Maselli, A. y Dierking, J. (2018). Influencia del CO2 disuelto en la medición de la Concentración en Bebidas Dietéticas. Parma: Maselli Misure.

Naddaf, L., Avalo, B., y Oliveros, M. (2012). Secado por aspersión de jugo natural de naranja utilizando los encapsulantes maltodextrina y goma arábiga. Revista Técnica de la Facultad de Ingeniería Universidad del Zulia, 35(1): 20-27.

Revelo, J., Viteri, P., Vásquez, W., Valverde, F., León, J., Gallegos, P. (2010). Manual del cultivo ecológico de la naranjilla. Quito: INIAP.

Silva, P., Durán, S. (2014). Bebidas azucaradas, más que un simple refresco. Revista Chilena de Nutrición, 41(1): 90-97.

Villareal, Y., Mejía, D., Osorio, O., y Cerón, A. (2013). Efecto de pasteurización sobre características sensoriales y contenido de vitamina $\mathrm{C}$ en jugos de frutas. Biotecnología en el Sector Agropecuario y Agroindustrial, 11(2), 66-75.

Viteri, P., Vásquez, W., León, F., Viera, W., Posso, M., Hinojosa, A., Revelo, M. Ochoa, J. (2009). INIAP Quitoense-2009 Naranjilla de jugo (Solanum quitoense Lam.) injerta en patrones de solanáceas silvestres resistentes a Fusarium oxysporum y a Meloidogyne incognita. Quito, Ecuador: INIAP. 